\title{
Type-IV Superconductivity: Cooper Pairs with Broken Inversion and Time-Reversal Symmetries in Conventional Superconductors
}

\author{
A.G. Lebed* \\ Department of Physics, University of Arizona, \\ 1118 E. 4-th Street, Tucson, AZ 85721, USA
}

\begin{abstract}
Vortex phase in a singlet superconductor in the absence of impurities is shown to be absolutely unstable with respect to the appearance of a triplet component which breaks both inversion and time-reversal symmetries of Cooper pairs. Symmetry breaking paramagnetic effects are demonstrated to be of the order of unity if the orbital upper critical field, $H_{c 2}(0)$, is of the order of Clogston paramagnetic limiting field, $H_{p}$. We suggest a generic phase diagram of such type-IV superconductor, which is singlet one at $H=0$ and characterized by mixed singlet-triplet order parameter with broken time-reversal symmetry in vortex phase. A possibility to observe type-IV superconductivity in clean organic, high- $\mathrm{T}_{c}, \mathrm{MgB}_{2}$, and other superconductors is discussed.
\end{abstract}

PACS numbers: 74.20.Rp, 74.25.Op, 74.70.-b 
It is well known that Meissner effect, which is the main feature of superconductivity phenomenon, is used to classify superconducting materials. In type-I superconductors, where Meissner effect is complete, superconductivity is destroyed at $H>H_{c}$ (where $H_{c}$ is thermodynamic critical field), whereas, in type-II superconductors, superconductivity phenomenon survives at highier magnetic fields, $H_{c 1}<H<H_{c 2}$ (where $H_{c 1}<H_{c}<H_{c 2}$ ) in the form of Abrikosov vortices $[1,2]$. Type-II superconductors can be subdivided into two main classes: superconducting alloys (or dirty superconductors) $[1,2]$ and relatively clean superconductors, where type-II superconductivity is due to anisotropy of their electron spectra and relatively high effective masses of quasi-particles [3]. Due to a success in synthesis of novel materials, a number of new classes of relatively clean type-II superconductors were discovered during last 30 years, including organic [4,5], heavy-fermion [6], high- $\mathrm{T}_{c}$ [7], $\mathrm{Sr}_{2} \mathrm{RuO}_{4}$ [8], $\mathrm{MgB}_{2}[9]$, and other superconductors. Currently, the above mentioned relatively clean type-II superconductors are the most interesting and important materials both from fundamental point of view and from point of view of their possible applications.

Usually, orbital superconducting order parameter, $\Delta\left(\mathbf{r}_{1}, \mathbf{r}_{2}\right)$, corresponding to pairing of two electrons in Cooper pair, can be expressed in the form $\Delta\left(\mathbf{r}_{1}, \mathbf{r}_{2}\right)=\Delta(\mathbf{R}) \hat{\Delta}(\mathbf{r})$. [Here, external order parameter, $\Delta(\mathbf{R})$, is related to motion of a center of mass of Cooper pair, $\mathbf{R}=\left(\mathbf{r}_{\mathbf{1}}+\mathbf{r}_{\mathbf{2}}\right) / 2$, whereas internal order parameter, $\hat{\Delta}(\mathbf{r})$, describes relative motion of electrons in Cooper pair, $\mathbf{r}=\mathbf{r}_{\mathbf{1}}-\mathbf{r}_{\mathbf{2}}$ ]. From this point of view, type-II superconductors in their vortex phases are characterized by broken symmetries of external order parameter, $\Delta(\mathbf{R})$, which is responsible for Meissner currents $[1,2]$.

Other important issues are symmetries of internal orbital order parameter, $\hat{\Delta}(\mathbf{r})$ (or its Fourier component $\hat{\Delta}(\mathbf{k}))$, and related spin part of superconducting order parameter, $\hat{\Delta}\left(\sigma_{1}, \sigma_{2}\right)$. In accordance with Fermi statistics, internal order parameter, $\hat{\Delta}(\mathbf{k})$, is an even function of variable $\mathbf{k}$ in the case of singlet superconductivity (where the total spin of Cooper pair $|\mathbf{S}|=0$ ), whereas $\hat{\Delta}(\mathbf{k})$ is an odd function of $\mathbf{k}$ in the case of triplet superconductivity (where $|\mathbf{S}|=1$ ) $[10,11]$. Depending on symmetry properties of $\hat{\Delta}(\mathbf{k})$, superconductors are subdivided into conventional ones [1,2] (where superconductivity can be described in terms of BCS s-wave singlet pairing) and unconventional ones $[10,11]$ (where symmetry of $\hat{\Delta}(\mathbf{k})$ is lower than the underlying symmetry of crystalline lattice).

It is commonly believed $[1,2,10,11]$ that magnetic field does not change internal superconducting order parameters (i.e., wave functions $\hat{\Delta}(\mathbf{k})$ and $\left.\hat{\Delta}\left(\sigma_{1}, \sigma_{2}\right)\right)$ and, thus, Cooper pairs can be considered as unchanged elementary particles in Abrikosov vortex phase. Moreover, although related to the external degrees of freedom Meissner currents break time-reversal symmetry of $\Delta(\mathbf{R})$, internal orbital and spin order parameters, $\hat{\Delta}(\mathbf{k})$ and $\hat{\Delta}\left(\sigma_{1}, \sigma_{2}\right)$, are believed to preserve $t \rightarrow-t$ symmetry. The main goal of our Letter is to show that there have to exist type-IV superconductors [12], which exhibit qualitatively different magnetic 
properties and are characterized by Cooper pairing with broken time reversal, $t \rightarrow-t$, and inversion, $\mathbf{k} \rightarrow-\mathbf{k}$, symmetries of internal order parameters in vortex phase. [Note that we define type-IV superconductivity as singlet superconductivity at $H=0$ and in Meissner phase which exhibits broken symmetries of Cooper pairs internal wave functions in vortex phase.]

More precisely, below we suggest and prove the following theorem: each singlet typeII superconductor in the absence of impurities is actually type-IV superconductor with broken $t \rightarrow-t$ and $\mathbf{k} \rightarrow-\mathbf{k}$ symmetries of internal order parameters in the vortex phase, provided that effective constant, responsible for triplet (p-wave) superconducting pairing, is not zero, $g_{t} \neq 0$. We show that the above mentioned theorem is due to careful account for paramagnetic spin splitting effects in vortex phase, which have been treated so far only in the case $g_{t}=0[1,2,10,11,13]$. In particular, we demonstrate that superconducting internal order parameter is a mixture of a singlet component, $\hat{\Delta_{s}}(\mathbf{k})$, with a triplet component, $i \hat{\Delta_{t}}(\mathbf{k})$, which breaks both inversion, $\mathbf{k} \rightarrow-\mathbf{k}$, and time-reversal, $t \rightarrow-t$, symmetries due to an imaginary coefficient $i$. We point out that the above mentioned effects of singlet-triplet mixing are expected to be of the order of unity in a number of modern relatively clean type-II superconductors, where $H_{c 2}(0) \sim H_{p}$ (see discussion in the end of the Letter). [Here, $H_{c 2}(0)$ is orbital upper critical field at $T=0[1,2,10,11]$ and $H_{p}$ is Clogston paramagnetic limiting field $[13,1]$.

Although, in the Letter, we consider vortex phase with broken symmetries only in Ginzburg-Landau (GL) region of a s-wave layered superconductor in a parallel magnetic field, we stress that the suggested theorem is very general and based only on symmetry arguments. As we argue below, the above mentioned theorem is a consequence of broken spin symmetry (due to paramagnetic spin splitting effects) [13] and broken translational symmetry of external orbital order parameter, $\Delta(\mathbf{R})$, in vortex phase. As a result, the theorem, suggested in the Letter, has to be valid for any s- and d-wave singlet superconductor [14] for both attractive and repulsive electron-electron interactions in a triplet ( $\mathrm{p}$-wave) channel.

In other words, the main our message is that Cooper pairs cannot be considered as unchanged elementary particles in a magnetic field in modern type-II superconductors, where $H_{c 2}(0) \sim H_{p}$. As shown below, magnetic fields of the order of $H \sim H_{p}$ qualitatively change the nature of Cooper pairs in vortex phase. We suggest that, in relatively clean conventional type-II superconductors, there have to exist the forth critical magnetic field, $H_{c 4}(T)$, corresponding to phase transition (or crossover) between Abrikosov vortex phase and exotic vortex phase, where inversion and time-reversal symmetries of Cooper pairs are broken and, thus, topologic properties of vortices are unusual (see Fig.1).

At first, let us qualitatively explain why paramagnetic effects lead to the appearance of a triplet component in vortex phase of a conventional singlet superconductor. It is well known 
$[10,11]$ that spin component of a singlet order parameter is antisymmetric function of spin variables, $\hat{\Delta}_{s}(+,-)=-\hat{\Delta}_{s}(-,+)$. In the presence of Abrikosov vortices, external order parameter, $\Delta(\mathbf{R})$, varies with coordinate $\mathbf{R}$, which corresponds to superconducting pairing of electrons with non-zero total momenta of Cooper pairs of the order of $|\mathbf{p}| \simeq \hbar / \xi$, where $\xi$ is a coherence length $[1,2,10,11]$. Let us consider superconducting pairing of two electrons with total momentum $\left|\mathbf{p}_{\mathbf{0}}\right| \neq 0$ in the presence of spin splitting paramagnetic effects (see Fig.2). As it is seen from Fig.2, absolute value of spin component $\Delta(+,-)$ is not equal to absolute value of spin component $\Delta(-,+)$ if $\left|\mathbf{p}_{\mathbf{0}}\right| \neq 0$. This means that $\Delta(+,-) \neq-\Delta(-,+)$ and, thus, in addition to singlet order parameter, a triplet component, corresponding to superconducting pairing with $|\mathbf{S}|=1$ and $S_{z}=0$, appears, where $S_{z}$ is a component of total spin of Cooper pair along magnetic field direction.

Below, we quantitatively describe superconducting pairing with internal order parameter, exhibiting broken inversion and time-reversal symmetries, in a singlet s-wave superconductor with layered electron spectrum,

$$
\epsilon_{0}(\mathbf{p})=\left(p_{x}^{2}+p_{y}^{2}\right) / 2 m+2 t_{z} \cos \left(p_{z} d\right), \quad \epsilon_{F}=m v_{F}^{2} / 2
$$

in a magnetic field:

$$
\mathbf{H}=(0, H, 0), \quad \mathbf{A}=(0,0,-H x) .
$$

In case, where electron-electron interactions do not depend on electron spins, the total Hamiltonian of electron system can be written as follows:

$$
\begin{aligned}
& \hat{H}=\hat{H}_{0}+\hat{H}_{i n t}, \quad \hat{H}_{0}=\sum_{\mathbf{p}, \sigma} \epsilon_{\sigma}(\mathbf{p}) c_{\sigma}^{+}(\mathbf{p}) c_{\sigma}(\mathbf{p}), \\
& \hat{H}_{i n t}=\frac{1}{2} \sum_{\mathbf{q}, \sigma} \sum_{\mathbf{p}, \mathbf{p}_{\mathbf{1}}} U\left(\mathbf{p}, \mathbf{p}_{\mathbf{1}}\right) c_{\sigma}^{+}\left(\mathbf{p}+\frac{\mathbf{q}}{2}\right) c_{-\sigma}^{+}\left(-\mathbf{p}+\frac{\mathbf{q}}{2}\right) c_{-\sigma}\left(-\mathbf{p}_{\mathbf{1}}+\frac{\mathbf{q}}{2}\right) c_{\sigma}\left(\mathbf{p}_{\mathbf{1}}+\frac{\mathbf{q}}{2}\right),
\end{aligned}
$$

where $\sigma= \pm 1, \epsilon_{\sigma}(\mathbf{p})=\epsilon_{0}(\mathbf{p})-\sigma \mu_{B} H, c_{\sigma}^{+}(\mathbf{p})$ and $c_{\sigma}(\mathbf{p})$ are electron creation and annihilation operators. As usually $[10,11]$, electron-electron interactions are subdivided into singlet and triplet channels:

$$
\begin{aligned}
& U\left(\mathbf{p}, \mathbf{p}_{\mathbf{1}}\right)=U_{s}\left(\mathbf{p}, \mathbf{p}_{\mathbf{1}}\right)+U_{t}\left(\mathbf{p}, \mathbf{p}_{\mathbf{1}}\right), U_{s}\left(\mathbf{p}, \mathbf{p}_{\mathbf{1}}\right)=U_{s}\left(-\mathbf{p}, \mathbf{p}_{\mathbf{1}}\right)=U_{s}\left(\mathbf{p},-\mathbf{p}_{\mathbf{1}}\right) \\
& U_{t}\left(\mathbf{p}, \mathbf{p}_{\mathbf{1}}\right)=-U_{t}\left(-\mathbf{p}, \mathbf{p}_{\mathbf{1}}\right)=-U_{t}\left(\mathbf{p},-\mathbf{p}_{\mathbf{1}}\right)
\end{aligned}
$$

Below, we define normal and anomalous (Gorkov) Green functions by standard way $[15,16]$ :

$$
\begin{aligned}
& G_{\sigma, \sigma}\left(\mathbf{p}, \mathbf{p}_{\mathbf{1}} ; \tau\right)=-<T_{\tau} c_{\sigma}(\mathbf{p}, \tau) c_{\sigma}^{+}\left(\mathbf{p}_{\mathbf{1}}, 0>, \quad F_{\sigma,-\sigma}\left(\mathbf{p}, \mathbf{p}_{\mathbf{1}} ; \tau\right)=<T_{\tau} c_{\sigma}(\mathbf{p}, \tau) c_{-\sigma}\left(-\mathbf{p}_{\mathbf{1}}, 0\right)>\right. \\
& F_{\sigma,-\sigma}^{+}\left(\mathbf{p}, \mathbf{p}_{\mathbf{1}} ; \tau\right)=<T_{\tau} c_{\sigma}^{+}(-\mathbf{p}, \tau) c_{-\sigma}^{+}\left(\mathbf{p}_{\mathbf{1}}, 0\right)>,
\end{aligned}
$$

where $<\ldots>$ stands for Gibbs averaging procedure with Hamiltonian (3). 
If we define singlet and triplet superconducting order parameters [10,11],

$$
\begin{aligned}
& \Delta_{s}(\mathbf{p}, \mathbf{q})=-\frac{1}{2} \sum_{\mathbf{p}_{1}} U_{s}\left(\mathbf{p}, \mathbf{p}_{\mathbf{1}}\right) T \sum_{\omega_{n}}\left[F_{+,-}\left(i \omega_{n} ; \mathbf{p}_{1}+\frac{\mathbf{q}}{2}, \mathbf{p}_{\mathbf{1}}-\frac{\mathbf{q}}{2}\right)-F_{-,+}\left(i \omega_{n} ; \mathbf{p}_{1}+\frac{\mathbf{q}}{2}, \mathbf{p}_{\mathbf{1}}-\frac{\mathbf{q}}{2}\right)\right] \\
& \Delta_{t}(\mathbf{p}, \mathbf{q})=-\frac{1}{2} \sum_{\mathbf{p}_{1}} U_{t}\left(\mathbf{p}, \mathbf{p}_{\mathbf{1}}\right) T \sum_{\omega_{n}}\left[F_{+,-}\left(i \omega_{n} ; \mathbf{p}_{1}+\frac{\mathbf{q}}{2}, \mathbf{p}_{\mathbf{1}}-\frac{\mathbf{q}}{2}\right)+F_{-,+}\left(i \omega_{n} ; \mathbf{p}_{1}+\frac{\mathbf{q}}{2}, \mathbf{p}_{\mathbf{1}}-\frac{\mathbf{q}}{2}\right)(6)\right.
\end{aligned}
$$

then, using Green-function technique $[10,11,15,16]$, we obtain the following equations:

$$
\begin{aligned}
& {\left[i \omega_{n}-\epsilon_{\sigma}(\mathbf{p})\right] G_{\sigma, \sigma}\left(i \omega_{n} ; \mathbf{p}, \mathbf{p}_{\mathbf{1}}\right)+\sum_{\mathbf{q}}\left[\sigma \Delta_{s}(\mathbf{p}, \mathbf{q})+\Delta_{t}(\mathbf{p}, \mathbf{q})\right] F_{-\sigma, \sigma}^{+}\left(i \omega_{n} ; \mathbf{p}-\mathbf{q}, \mathbf{p}_{\mathbf{1}}\right)=\delta\left(\mathbf{p}-\mathbf{p}_{\mathbf{1}}\right),} \\
& {\left[i \omega_{n}-\epsilon_{\sigma}(\mathbf{p})\right] F_{\sigma,-\sigma}\left(i \omega_{n} ; \mathbf{p}, \mathbf{p}_{\mathbf{1}}\right)-\sum_{\mathbf{q}}\left[\sigma \Delta_{s}(\mathbf{p}, \mathbf{q})+\Delta_{t}(\mathbf{p}, \mathbf{q})\right] G_{-\sigma,-\sigma}\left(-i \omega_{n} ;-\mathbf{p}_{\mathbf{1}},-\mathbf{p}+\mathbf{q}\right)=0} \\
& {\left[i \omega_{n}+\epsilon_{\sigma}(\mathbf{p})\right] F_{\sigma,-\sigma}^{+}\left(i \omega_{n} ; \mathbf{p}, \mathbf{p}_{\mathbf{1}}\right)+\sum_{\mathbf{q}}\left[\sigma \Delta_{s}^{+}(\mathbf{p}, \mathbf{q})+\Delta_{t}^{+}(\mathbf{p}, \mathbf{q})\right] G_{\sigma, \sigma}\left(i \omega_{n} ; \mathbf{p}+\mathbf{q}, \mathbf{p}_{\mathbf{1}}\right)=0,}
\end{aligned}
$$

which extend Gorkov equations $[15,16]$ to case of coexistence of singlet and triplet order parameters (6). [Note that Eqs.(7), suggested in the Letter, are rather general and describe coexistence of triplet and singlet order parameters for spin independent electron-electron interactions at arbitrary temperatures].

The goal of our Letter is to solve Eqs.(7) in the case, where layered superconductor (1) is placed in a parallel magnetic field (2). Below, we consider a phase transition line between metallic and singlet-triplet mixed superconducting phases in GL region (i.e., at $\left(T_{c}-T\right) / T_{c} \ll$ 1 ), where $T_{c}$ is a transition temperature from metallic state to s-wave singlet phase at $H=0$. For this purpose, we linearize $[17,10,11]$ Eqs.(7) with respect to superconducting order parameters, $\Delta_{s}(\mathbf{p}, \mathbf{q})$ and $\Delta_{t}(\mathbf{p}, \mathbf{q})$. As a result, we obtain the following system of linear equations:

$$
\begin{aligned}
& \Delta_{s}(\mathbf{p}, \mathbf{q})=-\frac{1}{2} \sum_{\mathbf{p}_{1}} U_{s}\left(\mathbf{p}, \mathbf{p}_{\mathbf{1}}\right) T \sum_{\omega_{n}}\left[\Delta_{s}\left(\mathbf{p}_{\mathbf{1}}, \mathbf{q}\right) S_{+}\left(i \omega_{n} ; \mathbf{p}_{\mathbf{1}}, \mathbf{q}\right)+\Delta_{t}\left(\mathbf{p}_{\mathbf{1}}, \mathbf{q}\right) S_{-}\left(i \omega_{n} ; \mathbf{p}_{\mathbf{1}}, \mathbf{q}\right)\right] \\
& \Delta_{t}(\mathbf{p}, \mathbf{q})=-\frac{1}{2} \sum_{\mathbf{p}_{1}} U_{t}\left(\mathbf{p}, \mathbf{p}_{\mathbf{1}}\right) T \sum_{\omega_{n}}\left[\Delta_{t}\left(\mathbf{p}_{\mathbf{1}}, \mathbf{q}\right) S_{+}\left(i \omega_{n} ; \mathbf{p}_{\mathbf{1}}, \mathbf{q}\right)+\Delta_{s}\left(\mathbf{p}_{\mathbf{1}}, \mathbf{q}\right) S_{-}\left(i \omega_{n} ; \mathbf{p}_{\mathbf{1}}, \mathbf{q}\right)\right] \\
& S_{+}\left(i \omega_{n} ; \mathbf{p}_{\mathbf{1}}, \mathbf{q}\right)=G_{+}^{0}\left(i \omega_{n}, \mathbf{p}_{1}+\frac{\mathbf{q}}{2}\right) G_{-}^{0}\left(-i \omega_{n},-\mathbf{p}_{1}+\frac{\mathbf{q}}{2}\right)+G_{-}^{0}\left(i \omega_{n}, \mathbf{p}_{1}+\frac{\mathbf{q}}{2}\right) G_{+}^{0}\left(-i \omega_{n},-\mathbf{p}_{1}+\frac{\mathbf{q}}{2}\right) \\
& \left.S_{-}\left(i \omega_{n} ; \mathbf{p}_{\mathbf{1}}, \mathbf{q}\right)\right]=G_{+}^{0}\left(i \omega_{n}, \mathbf{p}_{1}+\frac{\mathbf{q}}{2}\right) G_{-}^{0}\left(-i \omega_{n},-\mathbf{p}_{1}+\frac{\mathbf{q}}{2}\right)-G_{-}^{0}\left(i \omega_{n}, \mathbf{p}_{1}+\frac{\mathbf{q}}{2}\right) G_{+}^{0}\left(-i \omega_{n},-\mathbf{p}_{1}+\frac{\mathbf{q}}{2}(8)\right.
\end{aligned}
$$

where $G_{\sigma}^{0}\left(i \omega_{n}, \mathbf{p}\right)=1 /\left[i \omega_{n}-\epsilon_{\sigma}(\mathbf{p})\right]$ is Green function of a free electron in the presence of paramagnetic spin splitting effects. One of the main results of our Letter is that terms with $S_{-}\left(i \omega_{n}, \mathbf{p}_{1}, \mathbf{q}\right)$, mixing singlet and triplet superconducting pairings, are not equal to zero (see Fig.2). Therefore, in Abrikosov vortex phase, singlet component of superconducting order parameter always coexists with triplet one which breaks inversion symmetry of Cooper pairs.

As an example, let us consider coexistence of singlet s-wave and triplet p-wave order parameters, where

$$
U_{s}\left(\mathbf{p}, \mathbf{p}_{1}\right)=-\left(2 \pi / v_{F}\right) g_{s}, \quad U_{t}\left(\mathbf{p}, \mathbf{p}_{1}\right)=-\left(4 \pi / v_{F}\right) g_{t} \cos \left(\phi-\phi_{1}\right), \quad g_{s}>0, \quad g_{s}>g_{t}
$$


with $\phi$ and $\phi_{1}$ being polar angles corresponding to momenta $\mathbf{p}$ and $\mathbf{p}_{\mathbf{1}}$, respectively. [Note that inequalities $g_{s}>0$ and $g_{s}>g_{t}$ correspond to stabilization of a singlet $s$-wave superconductivity at $H=0$ ]. After substituting Eqs.(9) in Eqs.(8), we can represent singlet and triplet components of the order parameter as follows: $\Delta_{s}(\mathbf{p}, \mathbf{q})=\Delta_{s}(\mathbf{q})$ and $\Delta_{t}(\mathbf{p}, \mathbf{q})=\cos \phi \Delta_{t}(\mathbf{q})$, which satisfy the equations:

$$
\Delta_{s}(\mathbf{q})=g_{s} A \Delta_{s}(\mathbf{q})+g_{s} B \Delta_{t}(\mathbf{q}), \quad \Delta_{t}(\mathbf{q})=g_{t} C \Delta_{t}(\mathbf{q})+g_{t} D \Delta_{s}(\mathbf{q})
$$

Since z-component of vector potential (2) depends only on coordinate $x$, we may consider $q_{y}=0$ in Eqs.(10). Below, we calculate quantities $A, B, C$, and $D$ in GL region $[3,18,10,11]$ which corresponds to their expansions as power series in small parameters $v_{F} q_{x} / T_{c} \ll 1$, $t_{z} d q_{z} / T_{c} \ll 1$, and $\mu_{B} H / T_{c} \ll 1$. As a result, we obtain:

$$
\begin{aligned}
& A \simeq(\pi T) \sum_{\omega_{n}>0}^{\Omega}\left[\frac{2}{\omega_{n}}-\frac{1}{4 \omega_{n}^{3}}\left(v_{F}^{2} q_{x}^{2}+4 t_{z}^{2} q_{z}^{2} d^{2}+8 \mu_{b}^{2} H^{2}\right)\right], B \simeq-\sqrt{2} \mu_{B} H v_{F} q_{x}\left(\pi T_{c}\right) \sum_{\omega_{n}>0}^{\infty} \frac{1}{\omega_{n}^{3}}, \\
& C \simeq(\pi T) \sum_{\omega_{n}>0}^{\Omega}\left[\frac{2}{\omega_{n}}-\frac{1}{4 \omega_{n}^{3}}\left(3 v_{F}^{2} q_{x}^{2} / 2+4 t_{z}^{2} q_{z}^{2} d^{2}+8 \mu_{b}^{2} H^{2}\right)\right], D=B,
\end{aligned}
$$

where $\Omega$ is a cut-off energy.

We introduce magnetic field (2) in Eqs.(11) using a standard quasi-classical eiconal approximation $[17,18,10,11]: q_{x} \rightarrow-i(d / d x), q_{z} / 2 \rightarrow e A_{z} / c=e H x / c$, which leads to the following GL equations extended to the case of triplet-singlet coexistence:

$$
\begin{aligned}
& {\left[\tau+\xi_{\|}^{2} \frac{d^{2}}{d x^{2}}-\frac{\left(2 \pi \xi_{\perp}\right)^{2}}{\phi_{0}^{2}} H^{2} x^{2}\right] \Delta_{s}(x)+i \frac{\sqrt{7 \zeta(3)}}{\sqrt{2} \gamma} \xi_{\|}\left(\frac{H}{H_{p}}\right) \frac{d \Delta_{t}(x)}{d x}=0,} \\
& {\left[\frac{g_{t}-g_{s}}{g_{t} g_{s}}+\frac{3}{2} \xi_{\|}^{2} \frac{d^{2}}{d x^{2}}-\frac{\left(2 \pi \xi_{\perp}\right)^{2}}{\phi_{0}^{2}} H^{2} x^{2}\right] \Delta_{t}(x)+i \frac{\sqrt{7 \zeta(3)}}{\sqrt{2} \gamma} \xi_{\|}\left(\frac{H}{H_{p}}\right) \frac{d \Delta_{s}(x)}{d x}=0}
\end{aligned}
$$

where $\tau=\left(T_{c}-T\right) / T_{c} \ll 1, \xi_{\|}=\sqrt{7 \zeta(3)} v_{F} / 4 \sqrt{2} \pi T_{c}, \xi_{\perp}=\sqrt{7 \zeta(3)} t_{z} d / 2 \sqrt{2} \pi T_{c}$ are GL coherence lengths $[3,1,2], \phi_{0}$ is a flux quantum, $\zeta(z)$ is Riemann zeta-function, $\gamma$ is Euler constant, $H_{p}$ is Clogston paramagnetic limiting field [13].

In case, where $g_{s}-g_{t} \sim g_{s}$, Eqs. (12) have the following solutions:

$$
\Delta_{s}(x)=\exp \left(-\frac{\tau x^{2}}{2 \xi_{\|}^{2}}\right), \quad \Delta_{t}(x)=i \frac{\sqrt{7 \zeta(3)}}{\gamma}\left(\frac{g_{t} g_{s}}{g_{t}-g_{s}}\right) \sqrt{\tau}\left(\frac{H}{H_{p}}\right)\left(\frac{\sqrt{\tau} x}{\sqrt{2} \xi_{\|}}\right) \exp \left(-\frac{\tau x^{2}}{2 \xi_{\|}^{2}}\right)
$$

Eqs.(12),(13) are the main results of our Letter. They extend GL equations [3,1,2] and their famous Abrikosov solution for superconducting nucleus $[19,1,2]$ to the case $g_{t} \neq 0$. Eqs.(12),(13) directly demonstrate that Abrikosov solution [19,1,2] is absolutely unstable in 
the absence of impurities and, thus, singlet order parameter is always mixed with triplet one in vortex phase for both attractive, $-g_{t}<0$, and repulsive, $-g_{t}>0$, interactions in triplet (p-wave) channel. From Eqs.(12),(13), it also follows that triplet component breaks not only inversion symmetry, but also time reversal symmetry since $\Delta_{t}^{*}(x) \neq \Delta_{t}(x)$ due to imaginary coefficient $i$.

We hope that our results (8)-(13) open new area of research: theoretical and experimental studies of exotic vortex superconducting phases in singlet superconductors with their properties being even more unusual than that in the so-called unconventional superconductors $[10,11]$. We stress that type-IV superconductivity phenomenon, suggested in the Letter, is inherent and very common property of singlet superconductivity. In fact, we have shown that each s-wave [14] pure type-II superconductor is actually type-IV superconductor. The finite amount of impurities may result in the appearance of the forth critical field, $H_{c 4}(T)$, which may correspond to phase transition (or crossover) between phase, $H_{c 1}(T)<H<H_{c 4}(T)$, where broken symmetries of Cooper pairs do not exist (or marginal) and phase, $H_{c 4}(T)<H<H_{c 2}(T)$, where broken inversion and time-reversal symmetries are essential (see Fig.1). From Eqs.(13), it is directly seen that symmetry breaking triplet component is of the order of unity at low temperatures in such modern strongly correlated superconductors as organic, high- $\mathrm{T}_{c}, \mathrm{MgB}_{2}$, and some others, where $\left|g_{t}\right| \sim\left|g_{s}\right|$ and $H_{c 2}(0) \sim H_{p}$. In conclusion, we point out that spin splitting and broken translational symmetry effects have been studied in Refs.[20-23] in different context.

The author devote results, obtained in this Letter, to his wife Natalia, whose enormous support have given him a courage to set and to attack type-IV superconductivity problem.

* Also Landau Institute for Theoretical Physics, 2 Kosygina Street, Moscow, Russia.

[1] A.A. Abrikosov, Fundamentals of Theory of Metals (Elsevier Science Publisher B.V., Amsterdam, 1988).

[2] P.G. de Gennes, Superconductivity of Metals and Alloys (Westview Press, Advanced Book Program, Boulder, 1999).

[3] L.P. Gorkov and T.K. Melik-Barkhudarov, Zh. Eksp. Teor. Fiz. 4도 1493 (1963) [Sov. Phys. JETP, $\underline{18}, 1031$ (1964)] .

[4] D. Jerome, A. Mazaud, M. Ribault, and K. Bechgaard, J. Phys. (Paris) Lett. 41, L-95 (1980).

[5] T. Ishiguro, K. Yamaji, and G. Saito, Organic Superconductors (Second Edition, SpringerVerlag, Heidelberg, 1998).

[6] F. Steglich, J. Aarts, C.D. Bredl, W. Leike, D. Meschede, W. Franz, and H. Schafer, Phys. Rev. Lett. $\underline{43}, 1892$ (1979). 
[7] J.G. Bednortz and K. Muller, Z. Rev. B $\underline{64}$, 189 (1986).

[8] Y. Maeno, H. Hashimoto, K. Yoshida, S. Nishizaki, T. Fujita, J.G. Bednortz and F. Lichtenberg, Nature $\underline{372}, 532$ (1994).

[9] J. Nagamatsu, N. Nakagawa, T. Muranaka, Y. Zenitani, and J. Akimitsu, Nature $\underline{410}, 63$ (2001).

[10] M. Sigrist and K. Ueda, Rev. Mod. Phys. $\underline{63}, 239$ (1991).

[11] V.P. Mineev and K.V. Samokhin, Introduction to Unconventional Superconductivity (Gordon and Breach Science Publishers, Australia, 1999).

[12] We do not use term "type-III superconductivity" in order to distinguish between suggested in the Letter novel bulk phenomenon and a so-called surface superconductivity, which is characterized by critical field $H_{c 3}(T)[1,2]$.

[13] A.M. Clogston, Phys. Rev. Lett. $\underline{9}, 266$ (1962); B.S. Chandrasekhar, Appl. Phys. Lett. $\underline{1}, 7$ (1962).

[14] Type-IV superconductivity phenomenon in layered d-wave superconductors is considered in A.G. Lebed, Phys. Rev. Lett., submitted (2005).

[15] L.P. Gorkov, Zh. Eksp. Teor. Fiz. $\underline{34}, 735$ (1958) [Sov. Phys. JETP ㄱ, 505 (1958)].

[16] A.A. Abrikosov, L.P. Gorkov, and I.E. Dzyaloshinskii, Methods of Quantum Field Theory in Statistical Physics (Dover Publications, New York, 1963).

[17] L.P. Gorkov, Zh. Eksp. Teor. Fiz. 그, 833 (1959) [Sov. Phys. JETP 프, 593 (1960)].

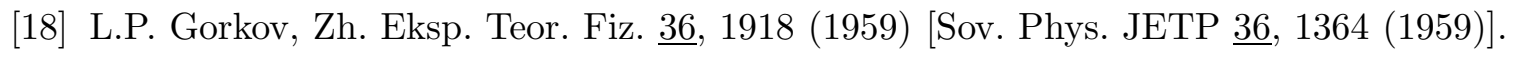

[19] A.A. Abrikosov, Zh. Eksp. Teor. Fiz. $\underline{32}, 1442$ (1957) [Sov. Phys. JETP $\underline{5}, 1174$ (1957)].

[20] M.M. Salomaa and G.E. Volovik, Rev. Mod. Phys. 모, 533 (1987).

[21] S. Matsuo, H. Shimahara, and K. Nagai, J. Phys. Soc. Jpn. $\underline{63}$, 2499 (1994).

[22] H. Shimahara, Phys. Rev. B $\underline{62}, 3524$ (2000).

[23] L.P. Gorkov and E.I. Rashba, Phys. Rev. Lett. 87, 037004 (2001). 


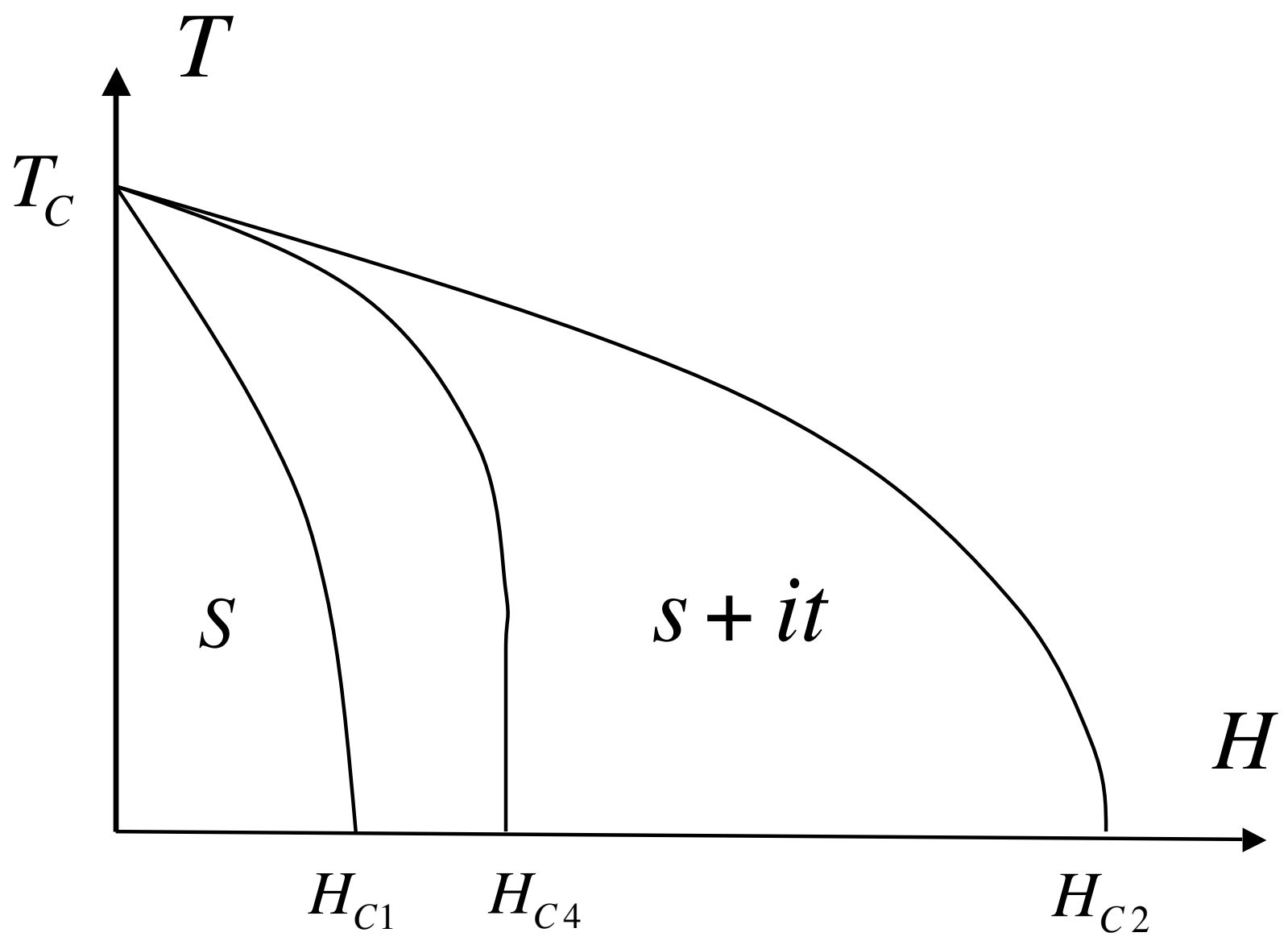

FIG. 1: A phase diagram of type-IV superconductor, which is singlet one at $H=0$ and in Meissner phase, $0<H<H_{c 1}(T)$, and characterized by broken inversion and time-reversal symmetries at low temperatures and high magnetic fields, $H_{c 4}(T)<H<H_{c 2}(T)$. In the intermediate region of magnetic fields, $H_{c 1}(T)<H<H_{c 4}(T)$, broken symmetries may be absent or marginal. 


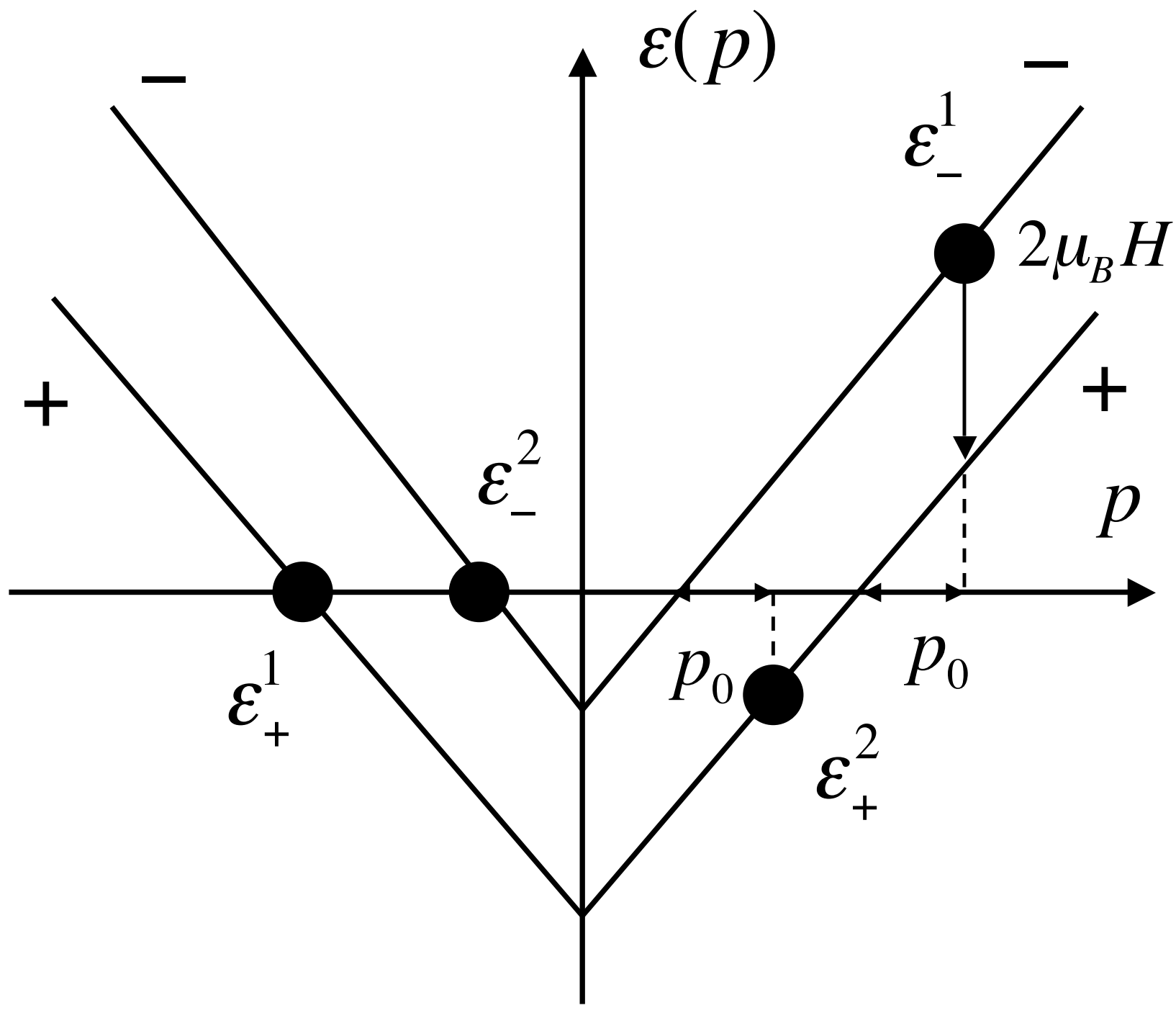

FIG. 2: In a magnetic field, electron spectra with spin up and spin down are split, $\epsilon_{+}(p)=$ $\epsilon_{0}(p)-\mu_{B} H$ and $\epsilon_{-}(p)=\epsilon_{0}(p)+\mu_{B} H$, respectively. Two Cooper pairs with spin parts of internal wave functions, $\Delta(+,-)$ and $\Delta(-,+)$, and total momenta $p_{0} \neq 0$ are characterized by different probabilities to exist since energy difference $\left|\epsilon_{+}^{1}-\epsilon_{-}^{1}\right|=p_{0} v_{F}+2 \mu_{B} H$ is not equal to energy difference $\left|\epsilon_{-}^{2}-\epsilon_{+}^{2}\right|=-p_{0} v_{F}+2 \mu_{B} H$ if $p_{0} \neq 0$. [For simplicity, linearized one-dimensional electron spectrum, $\epsilon(p)=v_{F}|p|$, is considered]. 